\title{
Adjacent Channel Interference in WCDMA Networks equipped with Multiple Antennas Mobile Stations
}

\author{
Julien Dumont \\ France Telecom R\&D \\ 38-40, rue du Général Leclerc \\ 92794 Issy-les-Moulineaux Cedex 9 \\ France \\ Email: julien.dumont@francetelecom.fr
}

\author{
Samson Lasaulce \\ CNRS - LSS \\ 5 , rue Joliot Curie \\ Plateau du Moulon \\ 91192 Gif-sur-Yvette \\ France \\ Email: lasaulce@1ss.supelec.fr
}

\author{
Jean-Marie Chaufray \\ France Telecom R\&D \\ 38-40, rue du Général Leclerc \\ 92794 Issy-les-Moulineaux Cedex 9 \\ France \\ Email: jeanmarie.chaufray@francetelecom.fr
}

\begin{abstract}
The purpose of this paper is to show to what extent multiple antenna terminals can combat inter-operator interference in WCDMA networks. To this end, a realistic modeling of adjacent channel interference - including filters imperfections and power amplifier non-linearities - is considered. Adjacent channel interference creates dead zones where the QoS target cannot be reached. In this paper, we first characterize these zones in terms of radius and probability of finding a given amount of users in it. In this respect, an interesting result is that the probability of finding one user in a dead zone can reach $48 \%$ for a $50 \%$ user load. But the main contribution is to show that certain linear multi-antenna reception schemes are able to cope with ACI. Compared to a conventional 1D Rake, the dead zone radius is decreased from $80 \mathrm{~m}$ to $20 \mathrm{~m}$ for typical NLOS scenarios. The results are far more significant when the interfering adjacent band operator has a LOS channel, the bit error rates is decreased from $50 \%$ to $0.1 \%$ around the interfering BS. At last, the best choice of the linear receiver scheme is discussed regarding propagation conditions (power delay profile, power angle profile, antenna correlation).
\end{abstract}

\section{INTRODUCTION}

In WCDMA cellular networks (e.g. UMTS - FDD) there are many sources of interference that are likely to degrade the mobile station (MS) performance. Interchip interference, interpath interference [1], multiple access interference, intercell interference and inter-system interference [2] are known to be the most influential sources of interference. However the adjacent channel interference (ACI) has not been deeply studied. In this paper we precisely focus on ACI. In the literature there are few papers presenting WCDMA inter-operator interference. In this respect [3] is a good reference. Where does ACI come from? ACI is due to the presence of several operators in the same geographical area. Adjacent band operators generate out-of-band emissions because of transmit filter imperfections and transmit power amplifier (PA) non-linearities. Additionally, the receiver filter has a finite frequency selectivity, which makes it sensitive to in-band emissions of adjacent band operators. As 3GPP specifications have been designed with cost and design constraints taken into account, there are situations where ACI can have a considerable impact on the receiver performance. The position of the mobile station within the main cell plays an important role regarding the influence of ACI. ACI power can be stronger than the useful signal when the mobile station of interest is very close to an adjacent band operator base station (BS) and distant from the useful BS. Note that ACI also exists in non-CDMA networks (e.g. GSM) but is not influential because frequency reuse is high (e.g. 7) while in CDMA networks it equals 1. Making a simple link budget analysis shows the existence of dead zones (DZ) in which the adjacent band operator blinds the mobile station under consideration.

In this paper we assume a network deployment based on $3 \mathrm{GPP}$ parameters. Because mobile stations are generally the limiting factor of cellular network performance, we only consider the downlink case. The proposed approach could be extended to the uplink. In this context, the ultimate goal of this paper is to know to what extent suited signal processing algorithms can compensate for transmit and receive devices imperfections. More specifically, we focus on low complexity multi-antenna reception algorithms. For the problem under consideration in this paper, namely ACI suppression in WCDMA networks, the most useful contributions on ACI cancellation are [4] and [5]. Both of these papers still focus on filters imperfections in TDMA systems and out-of-band emissions are taken into account. Additionally, single-antenna algorithms proposed in [4] rely on adjacent channels knowledge, which is not available in the considered problem. In [5], a useful array processing technique is proposed to combat ACI but would require major changes in order to be used to our context (CDMA, multipath channels, limited MS complexity).

Therefore the main purpose of this paper is fourfold:

- to consider a realistic modeling of adjacent channel interference, which essentially includes the PA non-linearity and filters imperfections;

- to characterize the importance of the dead zone problem (dead zone area, probability of finding a given number of users in a dead zone);

- to assess the impact of ACI on a conventional MS receiver (single-antenna, Rake receiver);

- to choose good multiple antenna reception schemes (in terms of the performance/complexity trade-off), evaluate the impact of using them for decreasing the dead zone radius and discuss the best scheme to be selected.

This paper is structured as follows. In section II we describe our signal model. Then we discuss in section III the importance of the dead zone problem. Section IV provides the considered multi-antenna reception algorithms. Corresponding simulation results are provided and discussed in section V. At last, in section VI we review the main results and contributions of this paper.

\section{PRoblem Statement}

In the paper we always consider two base stations: one useful base station (the subscriber's operator) and another one belonging to an adjacent band operator. As mentioned in the introduction, ACI stems both from the adjacent band BS out-of-band emissions and finite 
receive filter frequency selectivity. In the following two subsections we show how these imperfections can be taken into account in the signal model. We will introduce notations associated with the useful base station only and give the corresponding signal, since the adjacent band signal is generated in the same way up to a frequency offset ( $5 \mathrm{MHz}$ in the UMTS - FDD mode).

\section{A. Transmit signal model}

In this paper the downlink case is considered. No assumption is made on the number of antennas used by the useful base station. But we assume that the mobile station of interest is equipped with " $Q$ " sensors. Four sources of reception performance degradation are taken into account: the thermal noise, the interchip interference (ICI), the multiple access interference (MAI) and, of course, the adjacent channel interference generated by the adjacent band operator. To be more realistic, intercell should be accounted for. We will see that in the area of interest, which is in and around the dead zone, neglecting intercell interference does not prevent us from drawing useful interpretations from the presence of ACI in cellular networks.

Transmit filter output: denoting by $P_{k}$ the power allocated to user " $k$ ", $c_{k}(n)$ its spreading code, $b_{k}(n)$ its QPSK symbols, $s(n)$ the useful base station scrambling code and $g(t)$ the equivalent transmit filter allows us to express the baseband signal at the output of the transmit filter (fig. 1):

$$
x(t)=\sum_{n \in Z} \sum_{k=1}^{K} \underbrace{\sqrt{P_{k}} b_{k}(i) c_{k}(n) s(n)}_{d_{k}(n)} g\left(t-n T_{C}\right)
$$

where " $K$ " is the number of active users in the cell covered by the considered base station, " $i$ " is the symbol index, " $n$ " is the chip index and $T c$ is the chip duration. According to the UMTS-FDD mode specifications, the transmit filter is a root raised-cosine (RRC) filter with roll-off 0.22 and truncated to 8 chip durations (the purpose of this operation is to trade spectral performance against computational burden).

Power amplifier input: power amplification is the last stage of the transmitter, it has to amplify the in-phase plus in-quadrature signal (say $\tilde{x}(t)$ ), which writes as follows:

$$
\begin{aligned}
\tilde{x}(t) & =\tilde{x}_{I}(t)+\tilde{x}_{Q}(t) \\
& =\operatorname{Re}[x(t)] \cos \left(\omega_{0} t\right)+\operatorname{Im}[x(t)] \sin \left(\omega_{0} t\right)
\end{aligned}
$$

where $f_{0}=\omega_{0} / 2 \pi$ is the useful carrier frequency.

Power amplifier output: a good approximation of the power amplifier non-linearity is the polynomial model given in [6]. This approximation is often used by mobile and base stations manufacturers. Under this approximation, the PA output expresses as:

$$
y(t)=\tilde{x}(t) \sum_{i=0}^{D} a_{i}|\widetilde{x}(t)|^{2 i}
$$

where " $D$ " is the approximation degree and $\mathrm{a}_{0}=1$.

\section{B. Receive signal model}

Reception filter: the baseband filter is a discrete-time finite impulse response filter and is often chosen to be a RRC filter (roll-off 0.22). In WCDMA networks receiving mobile stations are sensitive to inband emissions of adjacent band operators because the latter is truncated (as in the transmitter) to 8 chip durations. Figure 2 shows the importance of truncating the RRC filter. Assuming a white input, it depicts the power spectral density (PSD) of the RRC output when the filter is truncated to 8 chip durations (top curve) and 32 chip durations (bottom curve). The out-of-band PSD levels differs by about $30 \mathrm{~dB}$

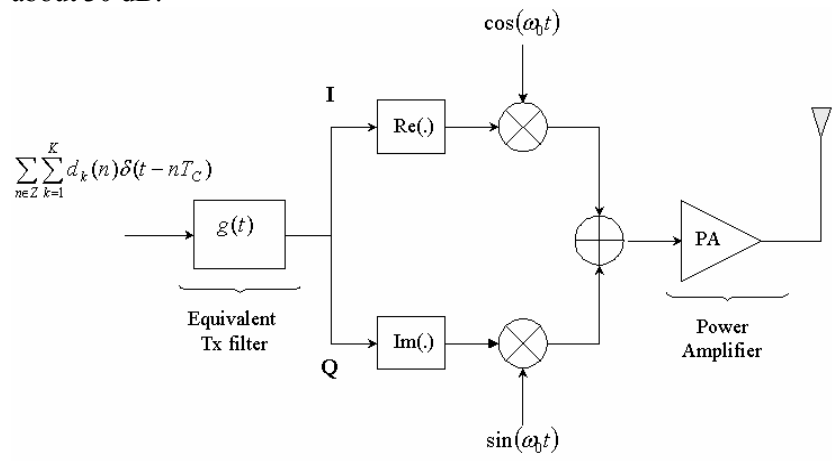

Figure 1: Transmitter model

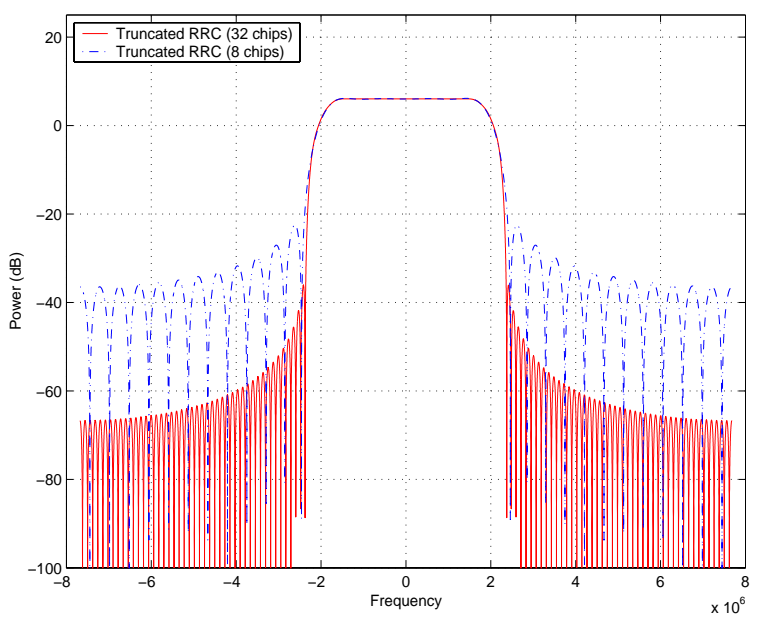

Figure 2: Influence of RRC truncation

Received signal model: although the received signal is generated through a non-linear device (PA), the receiver considers, for the baseband reception algorithms, a linear model of the transmission chain in the sense that the useful signal is separated from the ACI in an additive way. The discrete-time model that is used to the interference cancellation purpose is the following:

$$
\begin{aligned}
\underline{y}(n) & =\alpha_{u} \underbrace{\left(\sum_{k=1}^{K} \sum_{\ell=0}^{\ell=L-1} \underline{h}(\ell) d_{k}(n-\ell)\right)}_{\text {useful signal }+\mathrm{MAI}} \\
& +\alpha_{i} \underbrace{(\underbrace{(i n)}(n)+\underline{i}^{(\text {out })}(n))}_{A C I}+\underbrace{v(n)}_{A W G N}
\end{aligned}
$$

where $y(\mathrm{n})=\left[\begin{array}{llll}y_{1}(n) & \ldots & y_{\mathrm{Q}}(n)\end{array}\right]^{\mathrm{T}}$ is the signal received by the $Q$-sensor MS antenna, " $L$ " is the number of paths of the overall channel impulse response $\underline{h}(),. d_{k}($.$) is the useful chip sequence defined in (1),$ $\alpha_{u}$ corresponds to propagation losses over the useful link, $\alpha_{i}$ corresponds to propagation losses over the ACI link. At last, notations $\underline{i}^{\text {(in) }}$ (.) and $\underline{i}^{\text {(out) }}$ (.) stands for ACI received in and out of the theoretical MS frequency band $\left[f_{0}-(1+\rho) / T c, f_{0}+(1+\rho) / T c\right], \rho$ 
being the RRC roll-off factor. Figure 3 clearly shows what we mean by in-band and out-of-band interference.

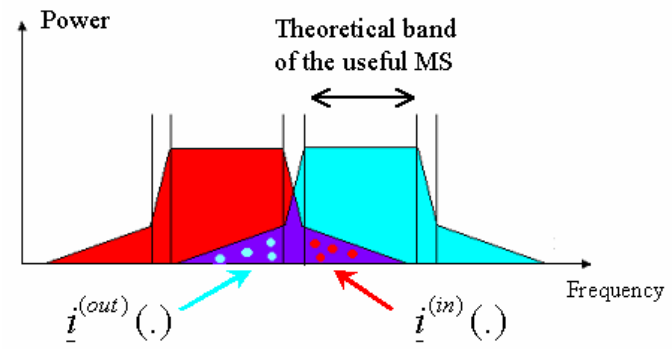

Figure 3: two origins of ACI

\section{Discussion On The IMPORTANCE OF The DEAD Zone EFFECT}

In the previous section we have seen how ACI can be modelled. The purpose of this section is to discuss the importance of ACI in cellular networks. We want to know how often the dead zone (DZ) phenomenon occurs. To this end we first review 3GPP's definition regarding ACI, then we give the propagation losses model we used in order to evaluate a typical dead zone radius. Making a simple analysis on the probability of the DZ phenomenon to occur concludes this section.

\section{A. Review of $3 G G P$ 's definitions on $A C I$}

Out-of-band-emissions maximum power: in the UMTS - FDD mode [7], the maximum level of base stations out-of-band emissions has been specified through the adjacent channel leakage ratio (ACLR). It is defined by $\operatorname{ACLR}_{\mathrm{BS}}=\mathrm{P}_{\mathrm{TX}}$ (in band) $/ \mathrm{P}_{\mathrm{TX}}$ (out-of-band) and has to be greater than $45 \mathrm{~dB}$.

Maximum of adjacent channel power: in the same way, the minimum frequency selectivity of receive mobile stations has been specified through the adjacent channel selectivity (ACS). It is defined by $\mathrm{ACS}_{\mathrm{MS}}=\mathrm{P}_{\mathrm{RX}}$ (in band) $/ \mathrm{P}_{\mathrm{RX}}$ (out-of-band) and has to be greater than 33 dB.

In order to measure the combined effects of transmission and reception in the adjacent band, the downlink adjacent carrier-tointerference ratio has been defined as $\mathrm{ACIR}^{-1}=\mathrm{ACLR}_{\mathrm{BS}}{ }^{-1}+\mathrm{ACS}_{\mathrm{MS}}{ }^{-1}$. The dominant part of $\mathrm{ACI}$ is due to MS frequency selectivity since $\mathrm{ACIR} \approx 32.7 \mathrm{~dB}$.

\section{B. Dead zone radius evaluation for a given SIR}

We first need to define more precisely what a dead zone is. In the downlink, it is an area around the adjacent band operator base station in which the QoS (quality of service) target cannot be reached. A dead zone can be roughly thought of as a circle centred on the adjacent band BS. This is why we allow ourselves to use the term "dead zone radius". Now, if we want to evaluate a dead zone radius we need to know the relation between the desired QoS and needed receive signal-to-total interference ratio (SIR), which is receiverdependent. In this subsection we assume that the required SIR is given. In practice the SIR target is obtained from desired QoS thanks to look-up tables. The chosen path loss model for the useful and ACI links is defined by:

$$
\left\{\begin{array}{l}
10 \log _{10}\left[\alpha_{u}\left(r_{u}\right)\right]=L_{u}+K_{u} \log _{10}\left(r_{u}\right) \\
10 \log _{10}\left[\alpha_{i}\left(r_{i}\right)\right]=L_{i}+K_{i} \log _{10}\left(r_{i}\right)
\end{array}\right.
$$

where $r_{u}$ and $r_{i}$ are the distance between the mobile station and the two base stations (useful and adjacent band BSs). If we assume that the ratio of the useful BS transmitted power to the transmitted ACI power is close to the standardized ACIR (target value), it is possible to easily relate the receive SIR condition to the condition on $\mathrm{r}_{\mathrm{i}}$. It can be checked that:

$$
\begin{aligned}
& \frac{P_{R X, u}}{P_{R X, i}} \geq S^{S I R_{d B}} \\
& \Rightarrow r_{i} \geq \underbrace{r_{u}^{K_{u} / K_{i}} \times 10^{K_{i}^{-1}\left(L_{u}-L_{i}+S I R_{d B}-A C I R_{d B}\right)}}_{R_{D Z}}
\end{aligned}
$$

Assuming a 3GPP typical NLOS urban micro-cell environment [8] for both the useful and ACI channels we find that the dead zone radius equals about 70 meters for a $0 \mathrm{~dB}$ SIR (voice service) and a $\mathrm{MS}$ at $500 \mathrm{~m}$ from the useful BS. In fact, this is not the worse case scenario since it can happen that the ACI propagation channel is less severe than the useful one and the SIR target can be higher than that (data service). In any case, compared to a typical micro-cell radius (about $600 \mathrm{~m}$ ), the DZ radius is significant even for low rates transmissions.

\section{Probability for active users to be in a dead zone}

Given a dead zone radius, we want to know what are the chances of one user (or more) to be in a dead zone. To this end we assume that the active users can be anywhere in the useful cell with an equal probability. We always denote by $K$ the number of users in the cell under consideration. Let $C$ be the number of users in a dead zone. We can show that the probability of the event $k \geq K_{0}$ to occur is roughly given by:

$$
\left\{\begin{array}{l}
P\left(k \geq K_{0}\right)=1-\sum_{i=0}^{K_{0}-1}\left(\begin{array}{c}
K_{0} \\
K
\end{array}\right) \varepsilon^{i}(K)[1-\varepsilon(K)]^{K-i} \\
\varepsilon(K)=\left(R_{D Z}(K) / R_{\text {cell }}\right)^{2}
\end{array}\right.
$$

In particular, one can check that when the number of active users goes to the infinity, the probability of finding one user in the DZ tends to 1 . Note that the dead zone radius depends on the user load because the QoS is MAI-dependent. For a 1D Rake, a 32 spreading factor and a $600 \mathrm{~m}$ cell radius we have obtained the following results:

\begin{tabular}{|c|c|c|c|c|}
\hline$K$ & 4 & 8 & 12 & 16 \\
\hline$R_{D Z}(m)$ & 60 & 70 & 80 & 120 \\
\hline$P(k \geq 1)$ & $4 \%$ & $10 \%$ & $19 \%$ & $48 \%$ \\
\hline
\end{tabular}

Table 1: Probability of finding 1 user in a DZ

Now we are convinced that what could be at a first glance considered as second-order effects can have a significant impact on the network performance, we propose studying different multi-antenna reception schemes in order to know to what extent signal processing can be of help to compensate for Tx and Rx imperfections.

\section{Multiple AntenNAs ReCEPTION SCHEMES}

We want to know to what extent using several antennas at the mobile station can help to combat ACI. In order to keep the receiver complexity reasonable we only study linear reception schemes. In this case the receiver consists of a linear space-time equalizer, followed by descrambling and despreading operations. One can show 
that for these reception strategies, the symbol estimate of the user of interest (index 1) has a generic expression:

$$
\hat{b}_{1}(i)=\sum_{n=0}^{N-1}\left[\sum_{l=0}^{L-1} \underline{w}^{H}(l) \underline{y}(n+l)\right] c_{1}(n) s^{*}(n)
$$

where $N$ is the spreading factor and $\underline{w}($.$) is the Q$-dimensional weighting vector depending on the reception scheme.

The best linear scheme is the chip-rate space-time Wiener filtering (STWF). This filter is designed in order to minimize the mean square error between its output and a reference sequence (the pilot sequence $d_{0}(n)$ for instance); it is given by

$$
\underline{w}=\left(E\left[\underline{Y}(n) \underline{Y}^{H}(n)\right]\right)^{-1} E\left[\underline{Y}(n) d_{1}^{*}(n)\right]
$$

where $\underline{w}$ and $\underline{Y}($.) are obtained by stacking the $L$ successive chip-rate samples $w(\ell)$ and $y(n+1), \ell=0, \ldots, L-1$, in a vector. In practice, this filter is implemented by replacing the expectation operator in (9) with a discrete sum over $n$. Hence, implementing the STWF requires the inversion of a $Q L \times Q L$ matrix. The complexity of this operation is too high regarding the fact that RRC filters have been truncated to decrease complexity. Additionally, the STWF performance can be severely degraded with respect to the strategies proposed below because of ill-conditioning of this matrix. In this context (limited complexity, continuous PAP, presence of a low-rank interference) the following reception schemes have been selected: conventional 2D RAKE, spatial matched filter (SMF), generalized 2D Rake and whitened 2D Rake.

\section{A. Conventional 2D Rake}

In this case there is one Rake per antenna and antenna outputs are simply added. It follows that:

$$
\underline{w}(\ell)=\underline{h}(\ell)
$$

It is known that this strategy is optimum when the noise-plusinterference term is Gaussian, temporally and spatially white.

\section{B. Generalized 2D Rake (G2D-Rake)}

The difference with the strategy above is that the outputs of each Rake are combined in order to minimize the chip estimate MMSE:

$$
\begin{aligned}
\underline{w}(\ell) & =\arg \min _{\underline{w}} E\left[\left\|\underline{w}^{H} \underline{z}(n)-d_{1}(n)\right\|^{2}\right] \\
& =E\left[\left(\underline{z}(n) \underline{z}^{H}(n)\right)^{-1}\right] E\left[\underline{z}(n) d_{1}^{*}(n)\right]
\end{aligned}
$$

where the vector $\underline{z}(n)=\left[z_{l}(n) \ldots z_{Q}(n)\right]^{\mathrm{T}}$ represents the outputs of the $Q$ Rake receivers.

\section{Spatial matched filter (SMF)}

A spatial filter matched to the pilot sequence is first applied to the received signal, and it is followed by a $1 \mathrm{D}$ RAKE receiver. The coefficients of the RAKE receiver are the spatially filtered versions of channel coefficients. For a given delay denoted by $\ell_{0}$, the weighting vector writes:

with

$$
\underline{w}(\ell)=\underline{a}\left(\ell_{0}\right) \underline{a}^{H}\left(\ell_{0}\right) \underline{h}(\ell)
$$

$$
\underline{a}\left(\ell_{0}\right)=\arg \min _{\underline{a}} E\left[\left|\underline{a}^{H} \underline{y}(n)-d_{1}\left(n-\ell_{0}\right)\right|^{2}\right]=\mathbf{R}_{y}^{-1} \underline{h}\left(\ell_{0}\right)
$$

and $\mathbf{R}_{y}^{-1}=E\left\lfloor\underline{y}(n) \underline{y}^{H}(n)\right\rfloor$. The delay $\ell_{0}$ is chosen in order to maximize:

$$
\underline{h}^{H}\left(\ell_{0}\right) R_{y}^{-1} \underline{h}\left(\ell_{0}\right)
$$

\section{Whitened 2D Rake (W2D-Rake)}

The idea here is to approximate space-time Wiener filtering by assuming the space-time covariance matrix to be block diagonal, which is equivalent to assume temporally white the noise-plusinterference term. It is easy to show that under this assumption the STWF boils down to:

$$
\underline{w}(\ell)=\mathbf{R}_{y}^{-1} \underline{h}(\ell)
$$

\section{Simulation Results}

Simulation setup: in all the simulations provided here, the number of active users is 8 , the spreading factor equals 32 and the useful channel is estimated thanks to the common pilot channel (2560 chips, $10 \%$ of the BS transmit power). The PA non-linearity is modelled by a 3-degree polynomial $\left(a_{1}=4 / 27\right)$ and works at its $1 \mathrm{~dB}$ compression point. By default, the useful and ACI channels correspond to a NLOS Vehicular A microcell environment [8], which corresponds to $L_{U}=L_{I}=34.53, K_{U}=K_{I}=38$. The corresponding power angle profiles are continuous and flat if nothing is specified. At last, the distance between useful and adjacent band operator base stations is $500 \mathrm{~m}$ by default and the mobile station is moving along a line between the useful and adjacent band base stations.

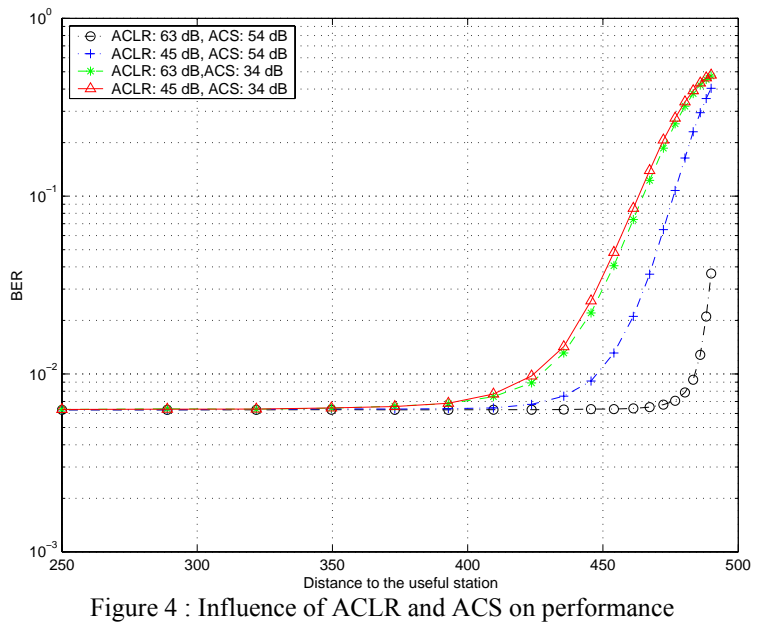

Dead zone existence: figure 4 represents the performance of a $1 \mathrm{D}$ Rake (raw BER) as a function of the distance in meter $\left(r_{u}\right)$ between the MS and serving BS. The top curve corresponds to what is obtained when using $3 \mathrm{GPP}$ specifications $\left(\mathrm{ACLR}_{\mathrm{BS}}=45 \mathrm{~dB}, \mathrm{ACS}_{\mathrm{MS}}=\right.$ $34 \mathrm{~dB}$ ). If the BER target is $1 \%$, the corresponding dead zone radius is about $80 \mathrm{~m}$. The other curves show what would be obtained if the Tx or/and Rx were improved in terms of ACLR or/and ACS. We also see that increasing the $\mathrm{ACS}_{\mathrm{MS}}$ significantly reduces the dead zone radius. We can notice that the receiver performance is the same between $250 \mathrm{~m}$ and $400 \mathrm{~m}$ : this is due to the 1D Rake error floor in presence of MAI, which makes it less sensitive to the link budget (or SNR).

Benefits of using multiple antennas at the MS: figure 5 depicts (from left to right) the performance of the 1D Rake (single antenna), 2D 
Rake, generalized 2D Rake, SMF, whitened 2D Rake. This time the MS is equipped with a 4-sensor linear array antenna (this assumption is made for simplicity but is not restrictive). Using the 2D Rake allows the DZ radius (BER target $=1 \%$ ) to be decreased from $80 \mathrm{~m}$ to $40 \mathrm{~m}$. The whitened 2D Rake achieves the best performance by reducing the DZ radius to $22 \mathrm{~m}$, which is remarkable. However one may ask about our assumption on the propagation environment (NLOS microcell) when the MS is very close to the interfering BS: what does the receiver performance become if a line-of-sight appears between the MS and interfering BS? The answer is given in the following subsection.

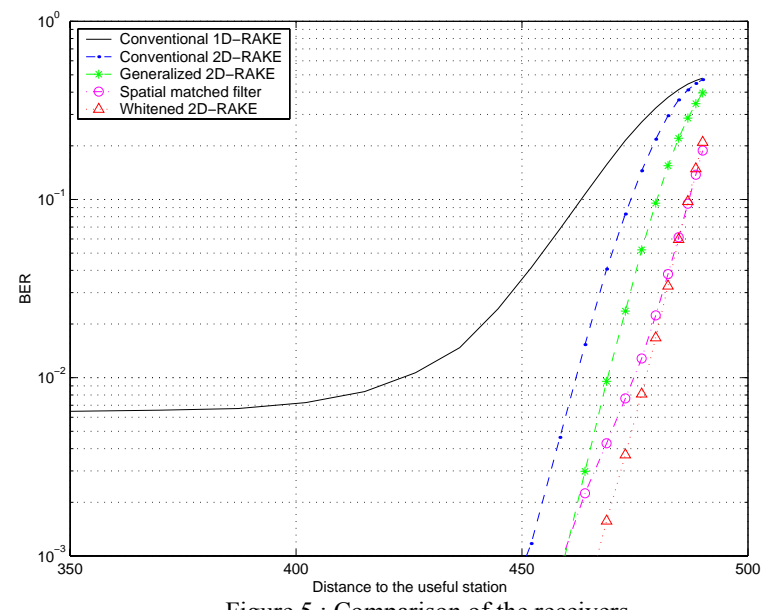

Figure 5 : Comparison of the receivers

Influence of the propagation environment: in order to compare the multi-antenna reception schemes between themselves, we provide in the table below raw bit error rates for different power delay profiles [8]. The mobile position is fixed and is close to the interfering station $(20 \mathrm{~m})$. In this case, the existence of a line-of-sight is possible. We first notice that the 1D-, 2D- and G2D-Rake performance are totally degraded by the presence of a line-of-sight, while SMF and W2DRake still get excellent performance. This can be explained by the fact that in the LOS case, there is only one dominant path coming from the interfering station. Indeed the presence of a LOS changes the interfering channel power angle profile, the latter becomes sparse and mobile sensors become correlated. It turns out that only SMF and W2D Rake are able to exploit this correlation to spatially reject the dominant interfering path whatever its power is. As for the comparison between SMF and W2D Rake we can see in the table that SMF is the best scheme when the interfering channel has few significant paths (like the Pedestrian A environment). On the other hand when the interfering channel becomes richer in terms of path (like Vehicular A), the W2D Rake is more suited.

\begin{tabular}{|c|c|c|c|c|c|c|}
\hline $\begin{array}{c}\text { Useful } \\
\text { Station }\end{array}$ & $\begin{array}{c}\text { Interfering } \\
\text { Station }\end{array}$ & $\begin{array}{c}\text { 1D } \\
\text { Rake }\end{array}$ & $\begin{array}{c}\text { 2D } \\
\text { Rake }\end{array}$ & $\begin{array}{c}\text { G 2D } \\
\text { Rake }\end{array}$ & SMF & $\begin{array}{c}\text { W 2D } \\
\text { Rake }\end{array}$ \\
\hline $\begin{array}{c}\text { Vehicular } \\
\text { A }\end{array}$ & $\begin{array}{c}\text { Vehicular } \\
\text { A }\end{array}$ & $32.6 \%$ & $21.8 \%$ & $9.5 \%$ & $2.2 \%$ & $1.7 \%$ \\
\hline $\begin{array}{c}\text { Vehicular } \\
\text { A }\end{array}$ & $\begin{array}{c}\text { Line-of- } \\
\text { Sight }\end{array}$ & $49.7 \%$ & $49.4 \%$ & $28.9 \%$ & $<0.1 \%$ & $0.2 \%$ \\
\hline $\begin{array}{c}\text { Pedestrian } \\
\text { A }\end{array}$ & $\begin{array}{c}\text { Pedestrian } \\
\text { A }\end{array}$ & $35.3 \%$ & $29.4 \%$ & $6.6 \%$ & $<0.1 \%$ & $<0.1 \%$ \\
\hline $\begin{array}{c}\text { Pedestrian } \\
\text { A }\end{array}$ & $\begin{array}{c}\text { Line-of- } \\
\text { Sight }\end{array}$ & $50 \%$ & $49.8 \%$ & $29.6 \%$ & $<0.1 \%$ & $<0.1 \%$ \\
\hline
\end{tabular}

Table 2: Influence of the PDP on the receiver performance

\section{CONCLUSIONS}

In this paper we have focused on a special kind of interference, namely ACI, not famous in the signal processing literature. Assuming a network deployment based on $3 \mathrm{GPP}$ parameters, we have provided simple but useful results to characterize the importance of the dead zone effect. We have showed how a dead zone radius can be found and the probability of finding a given number of users in it. In this respect having a $120 \mathrm{~m}$ dead zone and a $48 \%$ probability of finding one user in it is possible. Based on a realistic transmit and receive signals modeling we have showed that the dead zone effect plays an important role. We have seen that in the vicinity of the adjacent band base station, ACI is clearly the dominant interference and multiple access interference is no longer the limiting factor of the MS performance. In order to reduce this dramatic effect we have proposed to study adapted multi-antenna reception schemes taking into account MS complexity constraints. In this respect the spatial matched filter and whitened 2D Rake achieve the best performance. For typical scenarios using a 4-antenna SMF or W2D Rake allow the dead zone radius to be reduced from $80 \mathrm{~m}$ to $20 \mathrm{~m}$. We have also shown the influence of the propagation environment by evaluating the receiver performance for different power delay profiles and power angle profiles. An important result is that SMF and W2D Rake achieve very good performance $(0.1 \%$ bit error rates at 20 meters from the ACI base station) whatever the power angle (continuous or sparse) and power delay profiles are, which means that the proposed strategies work in any place of the network area (and then both for correlated and uncorrelated antennas). Several extensions of this study could be done. The uplink case could be considered. But what seems the most important work to be done is to make the general space-time Wiener filtering robust to ill-conditioning of the spacetime matrix to be inverted, which is important in the presence of lowrank interferers (like adjacent band interferers). The recent work of Honig and Goldstein [9] seems to be a good way of solving this problem.

\section{REFERENCES}

[1] An optimum combining and concatenated-Rake for dual-antenna mobile terminals in UMTS, S. Tantikovit and M. Wang, IEEE Comm. Letters, Vol 6, June 2002, Pages 231-233

[2] The effect of inter-system interference in UMTS at $1920 \mathrm{MHz}, \mathrm{H}$. Hass, S. MCLaughlin and G. Povey, IEE Conf. Publ. No 471, 2000, Pages 130-107.

[3] WCDMA inter-operator interference and dead zones, G. Povey, L. Gatzoulis, L. Stewart and I. Band, $5^{\text {th }}$ European Personal Mobile Communications Conference, April 2003, Glasgow, Scotland.

[4] Adjacent channel interference suppression in FDMA/TDMA mobile radio systems using joint demodulation, H. Arslan, S. Gupta, G. Bottomley and S. Cheunakeshu, ICC 1998, Vol 2, Pages 723-727.

[5] Adjacent channel interference rejection for land mobile radio systems, J. Ching Guey, A. Khayrallah and G. Bottomley, VTC 1998, Vol 3, Pages 1715-1719.

[6] Digital predistortion of wideband signals based on power amplifier model with memory, J. Kim and K. Konstantinou, Electronic Letters, November 2001, Vol 37, No 23.

[7] Evaluation of up- and downlink adjacent channel performance", Turin, 15-19 February 1999, TSGR4\#2(99)048, ,3GPP Technical Specification Group, Radio Access Network, Working Group 4

[8] 3GPP TS 25.996 v6.1.0 Technical Specification Group Radio Access Network; Spatial Channel Model for MIMO Simulations

[9] Adaptive reduced-rank interference suppression based on the multistage Wiener filter, M. Honig and J. Goldstein, IEEE Trans. on Communications, Vol 50, No 6, June 2002, Pages 986-994. 